\title{
Transition from order to chaos in molecular wave functions and spectra
}

\author{
F. J. Arranz and F. Borondo \\ Departamento de Química, C-IX, Universidad Autónoma de Madrid, Cantoblanco-28049, Madrid, Spain \\ R. M. Benito \\ Departamento de Física y Mecánica, E.T.S.I. Agrónomos, Universidad Politécnica de Madrid, 28040, \\ Madrid, Spain
}

(Received 17 January 1996; accepted 16 February 1996)

\begin{abstract}
In this Communication we describe how the transition from regularity to classical chaos in molecular Hamiltonian systems shows up at the quantum level in the structure of the corresponding wave functions and spectra. By changing the value of $\hbar$ we show how the scars result from combinations of regular wave functions. () 1996 American Institute of Physics. [S0021-9606(96)03316-6]
\end{abstract}

In the early stages of vibrational spectroscopy typical studies were only concerned with low lying vibrational states in which the nuclei move in a localized region around the minimum of the Born-Oppenheimer potential energy surface (PES). Anharmonic terms, responsible for the overtone and combination frequencies, were considered only as weak perturbations. ${ }^{1}$ In this regime, the intramolecular dynamics are completely regular and the spectra consist (at least in the ideal case) of a progression of bands, corresponding to the different excitations of each normal mode, that can easily be assigned, due to the lack of irregularities. The corresponding wave functions exhibit a very regular nodal pattern ${ }^{2}$ and quantum numbers can also, in principle, be assigned easily. Special care has to be exerted in the presence of classical resonances since they have a profound influence on the nodal structure of wavefunctions, as was demonstrated in the work of DeLeon, Davis, and Heller. ${ }^{3}$

At higher levels of excitation the dynamics of molecular systems change very much, and the interactions between normal modes ${ }^{4}$ cause the structure of the spectra to be more complicated. The KAM theorem dictates that more and more regular tori are destroyed as energy increases, rendering a multitude of resonant chains of islands, overlapping bands of stochasticity and embedded cantori. ${ }^{5}$ Nonlinear interactions among normal modes lead to irreversible intramolecular vibrational energy flow, which is controlled by all those classical structures. Also, the rate of many intramolecular processes, such as isomerization, unimolecular decomposition, etc., is determined by this intramolecular vibrational relaxation (IVR). ${ }^{6}$

Modern spectroscopy has broadened this horizon by the introduction of new techniques, such as IR overtone excitation, multiphonon excitation, stimulated emission pumping or electron photodetachment, ${ }^{7}$ in which extensive regions of the PES, sometimes very far from the equilibrium geometries, are probed. On the theoretical side, efficient methods have been developed to calculate accurately the eigenvalues and eigenfunctions of the vibrationally excited states involved. A special mention is due to those based on the discrete variable representation (DVR) method. ${ }^{8}$ In this energy regime, the interpretation of corresponding wave functions in simple terms is much more difficult. For moderate excitation energies choosing an adequate (curvilinear) coordinate system $^{9}$ can help. On the other hand, for very high vibrational energies some wave functions appear localized on periodic orbits (POs) of the system. This effect, known as "scarring", has received much attention in the literature ${ }^{10}$ although its origin is not yet fully understood. The POs of chaotic systems also determine the corresponding spectra, both at high ${ }^{11}$ and low resolution. ${ }^{12}$ The chaotic dynamics are very complex and only fully resolved when long times are considered; ${ }^{13}$ conversely if only low resolution is required merely the details of short trajectories or pieces of trajectories are needed. ${ }^{12,14}$

Time dependent methods have also witnessed a tremendous development in recent years. ${ }^{15}$ Wave packet propagation has also contributed greatly to our understanding of the relationship between spectra and the underlying dynamics. ${ }^{16}$

In this Communication, we discuss how the transition from order to classical chaos is reflected in the structure of the wave functions as energy increases. To illustrate this effect we use a 2D model for the molecular vibrations of the LiNC/LiCN system ${ }^{17}$ that has been extensively studied in the literature. In it, the $\mathrm{C}-\mathrm{N}$ distance is held constant at its equilibrium value, $r_{e}$, and the two remaining vibrational coordinates, $R$ and $\theta$, are defined as the distance from the $\mathrm{Li}$ atom to the $\mathrm{CN}$ center of mass and the angle between these two vectors, respectively. Motion in the bending angle is very floppy, sampling extensive regions of the PES. This is the origin of chaos in this system. The classical dynamics of this model can best be monitored by constructing Poincaré surfaces of sections (PSOS) using the minimum energy path (MEP), which connects the two linear isomers: LiNC $(\theta$ $\left.=180^{\circ}\right)$ and $\operatorname{LiCN}(\theta=0)$, as sectioning plane. ${ }^{18}$

In Fig. 1 a composite PSOS computed at an energy intermediate between those of the 24th and 25th eigenvalues is shown. At this energy the LiCN well is barely accessible (the first state located in this well is state number 31), and two very distinct regions on the LiNC can be distinguished: the inner one (around the elliptic fixed point marked with a star and corresponding to the stable isomer LiNC) which is regular, and the outer one that corresponds to chaotic motion. In this chaotic region we have also marked (with squares and triangles) two POs, stable and unstable, respectively, that 


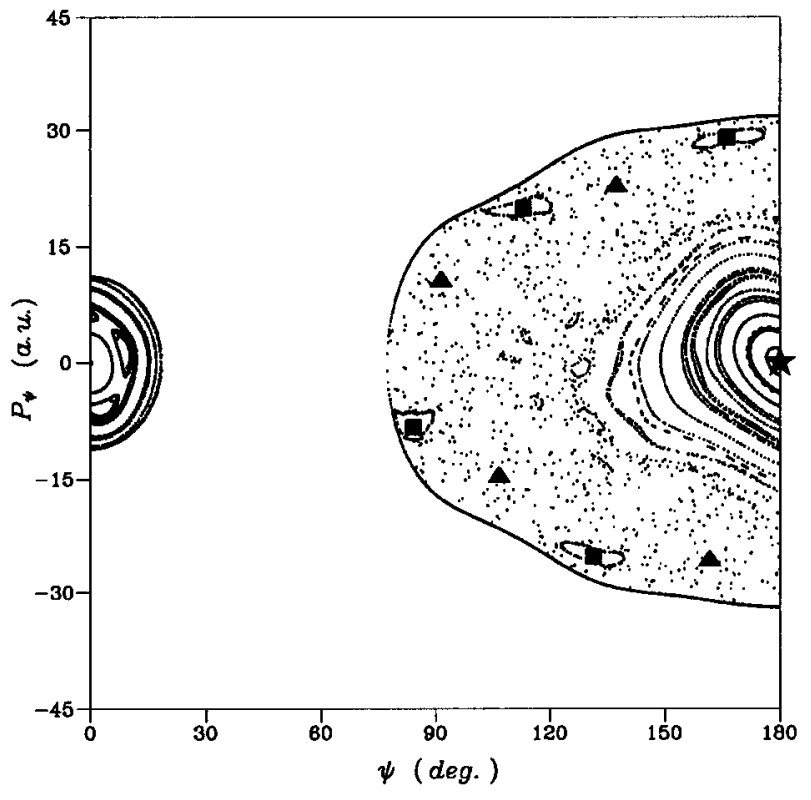

FIG. 1. Poincaré surface of section for the $\mathrm{LiNC} / \mathrm{LiCN}$ system at an energy of $2568.03 \mathrm{~cm}^{-1}$. Precise details on how the coordinates are defined can be found in Ref. 18. We have marked with a star the fixed elliptic point corresponding to the linear $\left(\psi=\theta=180^{\circ}\right)$ LiNC isomer, and with squares and triangles the elliptic and hyperbolic ones corresponding to a resonance relevant to the discussion presented in the text.

correspond to a resonant chain of islands which is relevant for the discussion below.

As energy increases the area of the regular region first increases due to the growth of the available phase space, and then starts to decrease due to the destruction of invariant tori dictated by the KAM theorem. As a result, up to a certain energy value the states have enough "phase space room to accommodate themselves" in the regular region and the increasingly excited bending states look regular, presenting a very clear nodal pattern. For energies above that value the bending states have a significant probability density extending into the chaotic region, and regular states with more excitation in the bending mode (approximately more than 22 quanta) are no longer found in the quantum calculations. ${ }^{19}$ In configuration space this would correspond to states trying to extend beyond the sharp curvature in the MEP (shown in the insets at the left of Fig. 2) taking place at $\theta=99^{\circ}$. This is in correspondance with the classical bobsled effect, ${ }^{20}$ by which trajectories coming from the LiNC well and passing beyond that point will inevitably pick up some excitation in the stretching mode on their way back. It is very difficult to make the present argument more quantitative due to the well known fact that quantum mechanics is somewhat "sluggish" in its response to classical chaos. ${ }^{21,3}$ Those states, very extended in $\theta$, present more complicated nodal patterns, and some of them are scarred along POs. For example, in the central part of Fig. 2 ( $\hbar=1$ a.u.) states 24 and 25 are shown, which appear scarred, respectively, by the unstable and stable POs marked with triangles and squares in Fig. 1.

As we show in this Communication, this transition from order to chaos in a realistic molecular system can be better

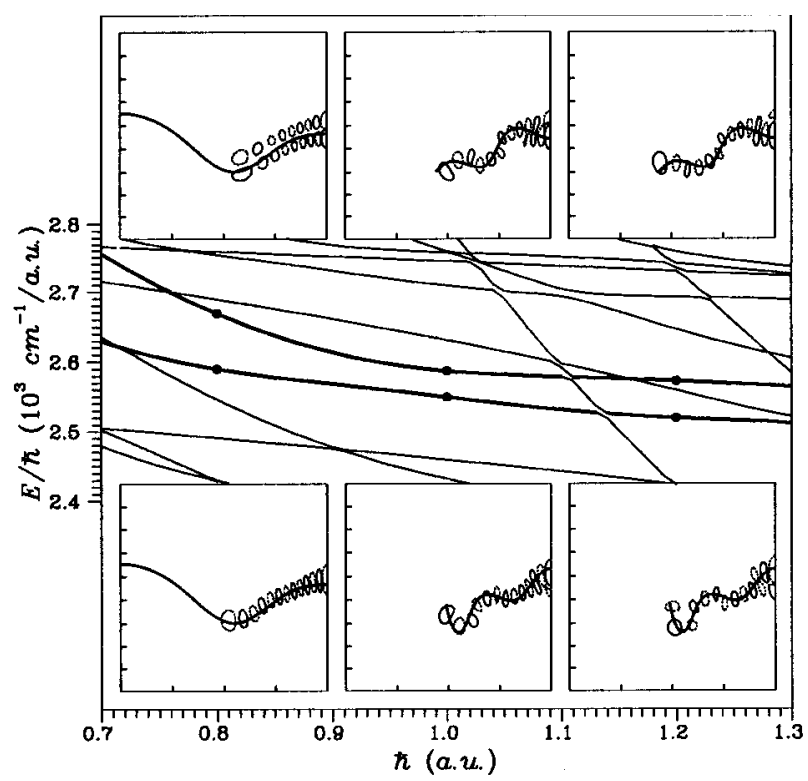

FIG. 2. Correlation diagram with $\hbar$ as parameter for several states of the LiNC/LiCN system. Notice that we have represented $E / \hbar$ vs $\hbar$ which renders horizontal lines in the harmonic limit. In the thicker line we have plotted the diabatic states corresponding to the 24th and 25th eigenstates of $\hbar=1$ a.u. The evolution of the corresponding wave functions for three selected values of $\hbar$ is also presented in the insets; in the horizontal axis the $\theta$ variable has been represented covering the range $0-180^{\circ}$, and the vertical one corresponds to $R$ over the range $2-6.5$ a.u. The wave functions for $\hbar=0.8$ a.u. are regular and have been plotted superimposed to the minimum energy path of the potential energy surface; the others are scarred by the unstable (lower insets) and stable (upper insets) periodic orbits marked in Fig. 1, which have been also represented in the figure.

understood if we consider calculations done by varying a parameter in our Hamiltonian. In our case changing $\hbar$ is most adequate since this parameter determines the number of states that the regular phase space region can accommodate. Results, calculated using the DVR method ${ }^{18}$ are shown in Fig. 2 where the energies (divided by $\hbar$ ) of several states as a function of $\hbar$ have been plotted. In this correlation diagram, it can be seen that the states represented in thicker line, which corresponds to states 24 and 25 at $\hbar=1$ a.u. and have been obtained by diabatizing ${ }^{22}$ the existing sharp avoided crossings, present a strong interaction (broad avoided crossing) between them at $\hbar \simeq 1$ a.u. As $\hbar$ decreases these states separate and consequently the interaction diminishes. The corresponding wave functions become regular, as can be seen in the insets on the left part of the figure, and show definite nodal patterns, which correspond in this case to $\left(n_{R}, n_{\theta}\right)$ quantum numbers of $(0,24)$ and $(1,16)$, respectively. These are some of the regular states that "cannot be found" in the usual ( $\hbar=1$ a.u.) quantum mechanical calculations. For $\hbar>1$ a.u. the nodal patterns get progressively more complicated and their interaction more important. From the correlation diagram of Fig. 2 it is clear that the scarred wave functions of states 24 and 25 are the result of the interaction and combination of the $(0,24)$ and $(1,16)$ regular states. We will provide more discussion on the actual values of the combination coefficients later in the paper.

The discussion presented above it is not only relevant in 


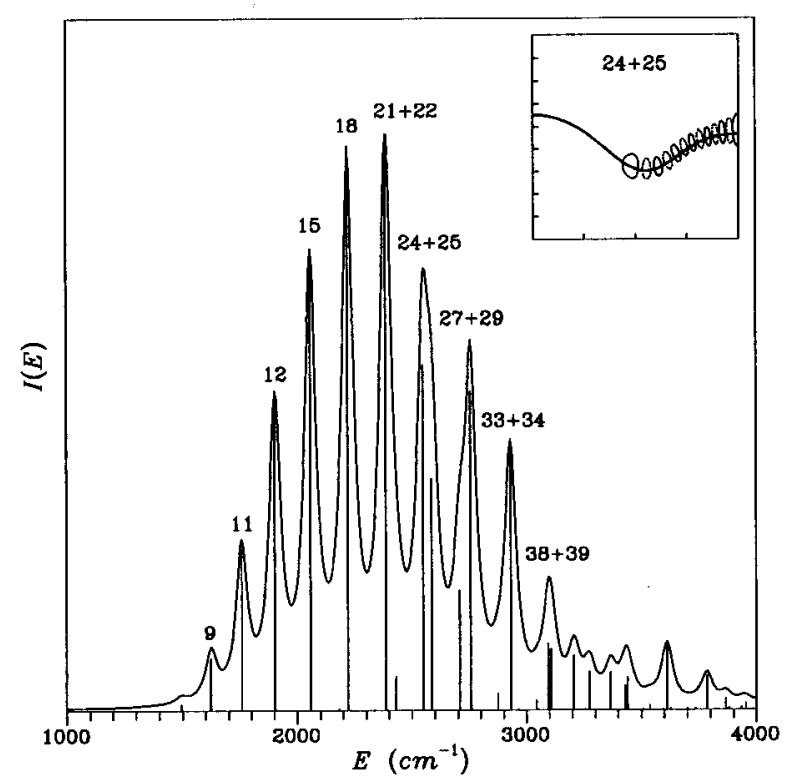

FIG. 3. Stick spectrum and low resolution version of it for a Gaussian wave packet initially centered on a point close to the minimum energy path: $(R, \theta)_{0}=\left(3.6\right.$ a.u., $\left.85^{\circ}\right)$. On top of each band the numbers of the contributing stationary states have been indicated. In the inset the wave function corresponding to the seventh band (see equation in the text) has been plotted superimposed to the minimum energy path; the axis ranges used are the same as in Fig. 2.

the context of theoretical quantum mechanical calculations of vibrational states, but it is also important for the discussion of the corresponding spectra. In order to illustrate this point we present in Fig. 3 the stick spectrum generated by a minimum uncertainty Gaussian wave packet (analogous to a stimulated emission pumping spectrum) initially located at $(R, \theta)_{0}=\left(3.6\right.$ a.u., $\left.85^{\circ}\right)$, a point approximately situated on the MEP. Superimposed on it we have also plotted a low resolution version resulting from the convolution with Lorentzian functions $60 \mathrm{~cm}^{-1}$ wide. It is seen that the low resolution spectrum is very simple and consists of a progression of ten bands, corresponding to transitions to states with increasing excitation on the bending mode, $\left(0, n_{\theta}\right)$, with $n_{\theta}$ even and starting from 12. On the top of each band the numbers of the contributing states have been indicated. The first five bands consist of just one state (which are all regular), and after that all the bands shown in the spectrum consist of two states. For example, the seventh band is the combination of the 24th and 25 th states. The wavefunctions associated to each band can be calculated by the simple projection technique proposed in Refs. 14 and 23, which only involves the calculation of the overlap Franck-Condon factors between the initial wave packet and the different eigenstates of the LiNC/LiCN system. The result for the seventh band that we are considering is that

$$
|\Psi\rangle=0.7726|24\rangle-0.6349|25\rangle \text {. }
$$

We show this wave function in the inset to Fig. 3, where it is clear that the band wave function is very regular, being highly localized on the MEP and corresponding to the $(0,24)$ diabatic state. Moreover, this band wave function is totally similar to the 24th eigenstate obtained at $\hbar=0.8$ a.u. which is shown in (the lower left corner of) Fig. 2. It is also clear from Fig. 2 that the diabatic state corresponding to the 25th eigenstate for $\hbar=0.8$ a.u. could be obtained by using a $p$-type (in the stretch coordinate) wave packet initially located at a slightly bigger value of $\theta_{0}$ to generate the spectrum.

Finally, we would like to emphasize that the procedure that we have suggested here to obtain a deeper insight in the relation between the transition from order to chaos and the scarring effect is not just a theoretical artifact. Varying the value of $\hbar$ in a quantum mechanical calculation is equivalent to scaling all masses of the problem. Although this is strictly not possible in a real molecule due to the reduced number of existing atomic isotopes, at least nature provides us with some combinations. For example in the present case of the $\mathrm{LiNC/LiCN}$ system, instead of the most abundant isotope combination ${ }^{7} \mathrm{Li}^{14} \mathrm{~N}^{12} \mathrm{C}^{7} \mathrm{Li}^{12} \mathrm{C}^{14} \mathrm{~N}$ used in the previous calculation, we can consider the alternative isotopic substitute ${ }^{9} \mathrm{Li}^{19} \mathrm{~N}^{16} \mathrm{C} /{ }^{9} \mathrm{Li}^{16} \mathrm{C}^{19} \mathrm{~N}$. For it we have found that the calculated 24th and 25th eigenfunctions (for $\hbar=1$ a.u.) look completely regular and correspond to the states $(0,24)$ and $(1,16)$, respectively.

Changing the atomic isotopes in a molecule can also introduce additional complications due to shifting of the vibrational frequencies and associated resonances; ${ }^{4}$ however, this is a point completely different from that raised in the present work.

To conclude, in this Communication we have shown how the transition from regularity to chaos that takes place in a realistic molecular Hamiltonian system is reflected in the structure of the corresponding wave functions. This can be accomplished by changing the value of $\hbar$, taken as a parameter of our model, or scaling the masses of the problem. As $\hbar$ decreases the system becomes more regular since more quantum states "can be accommodated" in the classical phase space region which is regular. By this procedure we have shown that it is the interaction and mixing of regular wave functions that generate the scars in the chaotic regime. The influence of this effect in the corresponding molecular spectra has also been discussed.

This work has been supported in part by DGICYT (Spain) under Contract No. PB92-0181. F.J.A. gratefully acknowledges a doctoral grant from the U.A.M.

${ }^{1}$ G. Herzberg, Molecular Spectra and Molecular Structure II. Infrared and Raman Spectra of Polyatomic Molecules (Van Nostrand, Princeton, 1964).

${ }^{2}$ R. M. Stratt, N. C. Handy, and W. H. Miller, J. Chem. Phys. 71, 3311 (1979).

${ }^{3}$ N. De Leon, M. J. Davis, and E. J. Heller, J. Chem. Phys. 80, 794 (1984).

${ }^{4}$ C. Jaffé and P. Brumer, J. Chem. Phys. 73, 5646 (1980); E. L. Sibert III, W. P. Reinhardt, and J. T. Hynes, J. Chem. Phys. 77, 3583 (1982).

${ }^{5}$ L. E. Reichl, The Transition to Chaos in Conservative Classical Systems: Quantum Manifestations (Springer-Verlag, New York, 1992).

${ }^{6}$ T. Uzer, Phys. Rep. 199, 75 (1991); S. A. Schofield, P. G. Wolynes, and R. E. Wyatt, Phys. Rev. Lett. 74, 3720 (1995).

${ }^{7}$ Laser Spectroscopy of Highly Vibrationally Excited Molecules, edited by V. S. Letokhov (Adams Hilger, New York, 1989); Molecular Dynamics and Spectroscopy by SEP edited by H. L. Dai and R. W. Field (World Scientific, Singapore, 1995)

${ }^{8}$ Z. Bacic and J. Light, Ann. Rev. Phys. Chem. 40, 469 (1989).

${ }^{9}$ D. T. Colbert and E. L. Silbert III, J. Chem. Phys. 91, 350 (1989). 
${ }^{10}$ E. J. Heller, in Chaos and Quantum Physics, edited by M. Giannoni, A. Voros, and J. Zinn-Justin (Elsevier, Amsterdam, 1991).

${ }^{11}$ M. C. Gutzwiller, Chaos in Classical and Quantum Mechanics (Springer, New York, 1990).

${ }^{12}$ J. M. Gomez-Llorente and E. Pollak, Ann. Rev. Phys. Chem. 43, 91 (1992); S. C. Farantos, J. M. Gomez-Llorente, O. Hahn, and H. S. Taylor, Chem. Phys. Lett. 166, 71 (1990).

${ }^{13}$ Remember the relation existing between spectrum and correlation function:

$I(E)=\Sigma|\langle\phi(0) n\rangle|^{2} \delta\left(E-E_{n}\right)=(1 / 2 \pi \hbar) \int d t\langle\phi(0) \mid \phi(t)\rangle e^{-i E t / \hbar}$.

${ }^{14}$ J. M. Gomez-Llorente, N. Berenger, F. Borondo, and R. M. Benito, Chem. Phys. Lett. 192, 430 (1992).

${ }^{15}$ C. Leforestier, R. Bisseling, C. Cerjan, M. D. Feit, R. Friesner, A. Guldberg, A. Hammerich, G. Jolicard, W. Karrlein, H. D. Meyer, N. Lipkin, O. Roncero, and R. Kosslofff, J. Comput. Phys. 94, 59 (1991).
${ }^{16}$ E. J. Heller, J. Chem. Phys. 62, 1544 (1975); R. Schienke, Photodissociation Dynamics (Cambridge University Press, Cambridge, England, 1992).

${ }^{17}$ F. Borondo and R. M. Benito, in Frontiers of Chemical Dynamics, edited by E. Yurtsever (Klewer, Dordrecht, 1995).

${ }^{18}$ R. M. Benito, F. Borondo, J. H. Kim, B. G. Sumpter, and G. S. Ezra, Chem. Phys. Lett. 161, 60 (1989).

${ }^{19} \mathrm{R}$. M. Benito and F. Borondo (in preparation).

${ }^{20}$ W. H. Miller, J. Chem. Phys. 72, 99 (1980).

${ }^{21}$ W. P. Reinhardt and C. Jaffé, Quantum Mechanics in Mathematics, Chemistry and Physics, edited by K. E. Gustavson and W. P. Reinhardt (Plenum, New York, 1981).

${ }^{22}$ A. Macias and A. Riera, Phys. Rep. 90, 81 (1982).

${ }^{23}$ G. G. de Polavieja, F. Borondo, and R. M. Benito, Phys. Rev. Lett. 73, 1613 (1994) 